AIAA-2000-1383

\title{
STRUCTURAL SIMILITUDE AND SCALING LAWS FOR PLATES AND SHELLS: A REVIEW
}

\author{
G.J. SIMITSES ${ }^{*}$ - University of Cincinnati \\ Cincinnati, Ohio 45221-0070 \\ J.H. STARNES, JR. ${ }^{+}$- NASA Langley Research Center \\ Mail Stop 190, Hampton, Virginia 23665 \\ J. REZAEEPAZHAND ${ }^{+}$- Ferdossi University of Mashhad \\ Mashhad, Iran
}

\begin{abstract}
This paper deals with the development and use of scaled-down models in order to predict the structural behavior of large prototypes. The concept is fully described and examples are presented which demonstrate its applicability to beam-plates, plates and cylindrical shells of laminated construction The concept is based on the use of field equations, which govern the response behavior of both the small model as well as the large prototype. The conditions under which the experimental data of a small model can be used to predict the behavior of a large prototype are called scaling laws or similarity conditions and the term that best describes the process is structural similitude. Moreover, since the term scaling is used to describe the effect of size on strength characteristics of materials, a discussion is included which should clarify the difference between "scaling law" and "size effect". Finally, a historical review of all published work in the broad area of structural similitude is presented for completeness.
\end{abstract}

\footnotetext{
- Professor of Aerospace Engineering, Fellow

${ }^{+}$Fellow

Assistant Professor \& Head of Mechanical Engineering.
}

Copyright (O 1999 by G.J. Simitses. Published by ALAA Inc, with permission.

\section{Introduction}

Aircraft and spacecraft comprise the class of aerospace structures that require efficiency and wisdom in design, sophistication and accuracy in analysis and numerous and careful experimental evaluations of components and prototype, in order to achieve the necessary system reliability, performance and safety.

Preliminary and/or concept design entails the assemblage of system mission requirements, system expected performance and identification of components and their connections as well as of manufacturing and system assembly techniques. This is accomplished through experience based on previous similar designs, and through the possible use of models to simulate the entire system characteristics.

Detail design is heavily dependent on information and concepts derived from the previous step. This information identifies critical design areas which need sophisticated analyses, and design and redesign procedures to achieve the expected component performance. This step may require several independent analysis models, which, in many instances, require component testing.

The last step in the design process, before going to production, is the verification of the design. This step necessitates the production of large components and prototypes in order to test component and system analytical predictions and verify strength and performance requirements under the worst loading conditions that the system is expected to encounter in service. 
Clearly then, full-scale testing is in many cases necessary and always very expensive. In the aircraft industry, in addition to full-scale tests, certification and safety necessitate large component static and dynamic testing. The C-141 A ultimate static tests include eight wing tests, 17 fuselage tests and seven empennage tests.' Such tests are extremely difficult, time consuming and definitely absolutely necessary. Clearly, one should not expect that prototype testing will be totally eliminated in the aircraft industry. It is hoped, though, that we can reduce full-scale testing to a minimum.

Moreover, crashworthiness aircraft testing requires full-scale tests and several drop tests of large components. The variables and uncertainties in crash behavior are so many that the information extracted from each test, although extremely valuable, is nevertheless small by comparison to the expense. Moreover, each test provides enough new and unexpected phenomena, to require new tests, specially designed to explain the new observations.

Finally, full-scale large component testing is necessary in other industries as well. Ship building, building construction, automobile and railway car construction all rely heavily on testing.

Regardless of the application, a scaled-down (by a large factor) model (scale model) which closely represents the structural behavior of the full-scale system (prototype) can prove to be an extremely beneficial tool. This possible development must be based on the existence of certain structural parameters that control the behavior of a structural system when acted upon by static and/or dynamic loads. If such structural parameters exist, a scaled-down replica can be built, which will duplicate the response of the fullscale system. The two systems are then said to be structurally similar. The term, then, that best describes this similarity is structural similitude.

\section{Bistorical Review}

Similarity of systems requires that the relevant system parameters be identical and these systems be governed by a unique set of characteristic equations. Thus, if a relation of equation of variables is written for a system, it is valid for all systems which are similar to it. $^{2}$ Each variable in a model is proportional to the corresponding variable of the prototype. This ratio, which plays an essential role in predicting the relationship between the model and its prototype, is called the scale factor. In establishing similarity conditions between the model and prototype, two procedures can be used, dimensional analysis and direct use of governing equations.

Models, as a design aid, have been used for many years, but the use of scientific models which are based on dimensional analysis was first discussed in a paper by Rayleigh. ${ }^{3}$ Similarity conditions based on dimensional analysis have been used since Rayleigh's time (Macagno ${ }^{4}$ ), but the applicability of the theory of similitude to structural systems was first discussed by Goodier and Thomson ${ }^{5}$ and later by Goodier. ${ }^{6}$ They presented a systematic procedure for establishing similarity conditions based on dimensional analysis.

There exist several books that refer to all elements of structural similitude. Murphy ${ }^{7}$, Langhaar, Charlton $^{9}$, Pankhurst ${ }^{10}$ and Gukhman ${ }^{11}$ all dcalt with similitude and modeling principles, and most of them dealt with dimensional analysis. $\mathrm{Kline}^{2}$ gives a perspective of the method based on both dimensional analysis and the direct use of the government equations. Szucs ${ }^{12}$ is particularly thorough on the topic of similitude theory. He explains the method with emphasis on the direct use of the governing equations of the system. A recent book by Singer, Arbocz and Weller $^{\text {if }}$ devotes an entire chapter on modeling with emphasis on dimensional analysis concepts.

A few studies concerning the use of scaleddown shell models have been conducted in the past. Ezra ${ }^{14}$ presented a study based on dimensional analysis, for buckling behavior subjected to impulse loads. A similar investigation was presented by Morgen ${ }^{15}$ for an orthotropic cylindrical shell subjected to a variety of static loads. Soedel ${ }^{16}$ investigated similitude for vibrating thin shells.

Due to special characteristics of advanced reinforced composite materials, they have been used extensively in weight efficient aerospace structures. Since reinforced composite components require extensive experimental evaluation, there is a growing interest in small scale model testing. Morton" discusses the application of scaling laws for impactloaded carbon-fiber composite beams. His work is based on dimensional analysis. Qian et al. ${ }^{18}$ conducted experimental studies of impact loaded composite plates, where the similarity conditions were obtained by considering the governing equations of the system. These works and many other experimental investigations have been conducted to characterize the size effect in material behavior for inelastic analysis (size effects are discussed in a later section).

In recent years, due to large dimensions and uniaue structural design of the proposed space station, 
small scale model testing and similitude analysis have been considered as the only option in order to gain experimental data. Shih et al. ${ }^{19}$, Letchworth et al. ${ }^{20}$, Hsu et al. ${ }^{21}$ and McGowan et al. ${ }^{22}$ discussed the possibility of scale model testing of space station geometries, especially for vibration analysis. Most of these studies have used complete similarity (defined in a later section) between model and prototype.

The present authors have published several papers (Simitses and Rezaeepazhand ${ }^{3}$, Rezaeepazhand et $a_{1}{ }^{24}$, Rezaeepazhand et $a^{25}{ }^{25}$ Simitses and Rezaeepazhand ${ }^{25}$. Simitses et al. ${ }^{27}$, and Rezaeepazhand and Simitses ${ }^{28}$ ) that deal with the design of scaled-down models and the use of test data of these models to predict the behavior of large prototypes. The behavior includes displacements, stresses, buckling loads, and natural frequencies of laminated beam-plates, plates and shells. In these studies, in the absence of model test data, the authors theoretically analyzed the models, and they used the similarity conditions, obtained by the use of the governing equations, to predict the behavior of the prototype. They then theoretically analyzod the prototype and they compared these results to the predictions. In most cases, the compared results were very close to each other and they concluded that the designed model can accurately predict the behavior of the prototype. Very recently, Ochoa and her collaborators ${ }^{29,30}$ applied similitude theory to a laminated cylindrical tube under tensile, torsion and bending loads and under external and internal pressure. They demonstrated the validity of developing a scale model, testing it and use the similarity conditions to predict the behavior of the prototype.

\section{Scaling Effects in Composites}

Considerable renewed interest has been exhibited in the broad field of scaling in the recent years, as evidenced by the multitude of research papers that have appeared in the technical literature. Before discussing any and all efforts, we must have a good understanding, for clear discussion of the meaning of the words that have been used. These words are scaling or scale effects, similarity conditions or scaling laws and size effects.

Scaling effects mean the effect of changing the geometric dimensions of a structure or structural component on the response to external causes. The external causes include all types of forces. Examples of the above is a beam made out of metallic material or man-made composite and subjected to bending. The main questions associated with predicting the response of the beam are: Are stiffness and strength affected by scaling? This means is the effective Young's modulus (both in tension and compression), which is usually obtained from small specimens affected by scale. In addition, is the strength affected by scale? Recognizing that beams are primarily designed for strength, the answer to the second question is important. On the other hand, since columns are primarily designed for stiffness (buckling), the answer to the first question is important.

In this context, the use of the term size effect is similar to the term scale effect. On the other hand, one may wish to find the conditions under which the behavioral response of a small size beam and a large size beam are similar. In this case, the interest is to find the similarity conditions or scaling laws in order to achieve similarity in response. In this context, the primary interest is to be able to test a small scale model, obtain response characteristics (displacements, buckling loads, vibration frequencies, etc.) and use the scaling laws to predict the behavior of the large prototype. In this second case, one can still use the term scaling effects, if he clearly does not refer to size effects on strength and stiffiness.

\section{Size Effects}

There exist two main sources of recent studies of size effects. First, Ref. 31 contains an outline of papers presented at a Workshop on Scaling Effects in Composite Materials and Structures, and second, Ref. 32 is a compilation of papers dealing with, primarily, fracture scaling.

From the conclusions, of virtually all presenters at the workshop ${ }^{31}$ who dealt with size effects, one can say that the size effect on stiffness is almost nonexistent. ${ }^{33,34,35}$ Similarly, the size effect on strength has created some controversy. Jackson ${ }^{33}$ concludes that there is considerable size effect on strength. Grimes ${ }^{36}$ contends that for solid laminates, the largest size effect on static strength is less than 4.5\%. Furthermore, he states that the cause of scale effects is not size but other factors such as poor quality tooling, differences in environmental exposure, etc. A similar conclusion was reached by $\mathrm{O}^{\prime} \mathrm{Brien}^{37}$ who claims that the effect of scaling is not because of size, but because different damage sequence occurs in two different sizes. In a private communication by L.B. Greszczuk $^{38}$ of McDonnell Douglas Space Systems Co., he stated, quote "If the small and big parts are made by the same process, there is not size effect neither on stiffiness nor on strength." He further explained that the tests performed at his company on specimens with twelve to one ratio in thickness (laminates), reveal that the effect on stiffness is nonexistent, while the effect on strength is less than $4 \%$. The specimens used were 
carefully manufactured by the same process and they had the same filament volume fraction and porosity.

The objective of most papers in Ref. 32 is to study the size effect on fracture of ice, concrete and notched composite beams. In these papers, the conclusion is that size does affect fracture and crack propagation.

One particular paper in Ref. 32, that by Daniel and $\mathrm{HsiaO}^{39}$, dealt with the thickness effect on compressive strength of unnotched laminates. It is an experimental study that used various sizes and layups and it concluded that the size effect is extremely small. Further evidence that size has negligible effect on stiffness is provided by the tests performed by Jackson ${ }^{40}$ on graphite/epoxy beams at NASA Langley. The scale varied from one-sixth to full and she employed unidirectional and quasi-isotropic layups.

Clearly, then one can at this junction say with confidence that size effect on stiffness is negligibly small and that more work on strength needs to be done in order to explain the reasons for the conflicting conclusions (if there is an effect, what causes it).

In view of the above, the authors embarked into a research program on structural similitude based on the following premises: (a) both model and prototype are governed by the same field equations (equilibrium, kinematic relations and constitutive equations, subject to boundary conditions), (b) the only set of equations that may be affected by size are the constitutive relations. It has already been concluded though that stiffiness is not affected by size and therefore one is safe to use the same constitutive relations for model and prototype up to but not in the vicinity of strength limits, (c) damage accumulation for both model and prototype is minimal. On this basis one can use similitude theory and obtain the similarity conditions.

\section{Theory of Similitude}

Similitude theory is concerned with establishing necessary and sufficient conditions of similarity between two phenomena. Establishing similarity between systems helps to predict the behavior of a system from the results of investigating other systems which have already been investigated or can be investigated more easily than the original system. Similitude among systems means similarity in behavior in some specific aspects. In other words, knowing how a given system responds to a specific input, the response of all similar systems to similar input can be predicted.

The behavior of a physical system depends on many parameters, i.e. geometry, material behavior, dynamic response and energy characteristics of the system. The nature of any system can be modeled mathematically in terms of its variables and parameters. A prototype and its scale model are two different systems with similar but not necessarily identical parameters. The necessary and sufficient conditions of similitude between prototype and its scale model require that the mathematical model of the scale model can be transformed to that of the prototype by a biunique mapping or vice versa $\left(\right.$ Szucs $\left.^{12}\right)$. It means, if vectors $X_{p}$ and $X_{m}$ are the characteristic vectors of the prototype and model, then we can find a transformation matrix $\Lambda$ such that:

$$
X_{p}=\Lambda X_{\text {. or }} X_{m}=\Lambda^{-1} X_{p}
$$

The elements of vector $\mathbf{X}$ are all the parameters and variables of the system. A diagonal form of the transformation matrix $\Lambda$ is the simplest form of transformation. The diagonal elements of the matrix are the scale factors of the pertinent elements of the characteristic vector $\mathbf{X}$

$$
\Lambda=\left[\begin{array}{llll}
\lambda \times 1 & 0 & \cdots & 0 \\
0 & \lambda \times 2 & \cdots & 0 \\
\vdots & \vdots & \ddots & \vdots \\
0 & 0 & \cdots & \lambda \times n
\end{array}\right],
$$

where $\lambda_{x_{i}}=x_{i} / x_{m}$ denotes the scale factor of $x_{i}$. In general the transformation matrix is not diagonal.

In establishing similarity conditions between the model and prototype two procedures can be used, dimensional analysis and direct use of governing equations. The similarity conditions can be established either directly from the field equations of the system or, if it is a new phenomenon and the mathematical model of the system is not available, through dimensional analysis. In the second case, all of the variables and parameters, which affect the behavior of the system, must be known. By using dimensional analysis, an incomplete form of the characteristic equation of the system can be formulated. This equation is in terms of nondimensional products of variables and parameters of the system. Then, similarity conditions can be established on the basis of this equation. 
In our studies, we consider only direct use of the governing equations procedure. This method is more convenient than dimensional analysis, since the resulting similarity conditions are more specific. When governing equations of the system are used for establishing similarity conditions, the relationships among variables are forced by the governing equations of the system.

The field equations of a system with proper boundary and initial conditions characterize the behavior of the system in terms of its variables and parameters. If the field equations of the scale model and its prototype are invariant under transformation $\Lambda$ and $\Lambda^{-1}$, then the two systems are completely similar. This transformation defines the scaling laws (similarity conditions) among all parameters, structural geometry and cause and response of the two systems. Examples of the direct use of governing equations is offered below.

\section{Bending of Laminated Beam-Plates}

Consider a laminated beamplate of length a and width $b$ and simply supported at both ends. We desire to find the maximum deflection of this beamplate. The beamplate is subjected to a transverse line load. By assuming that the displacement functions are independent of $y$, or $u=u(x), v=0, w=w(x)$ (cylindrical bending), from Ashton and Whitney ${ }^{41}$, the governing differential equations and boundary conditions are reduced to:

$$
\begin{aligned}
& \frac{d^{4} w}{d x^{4}}=\frac{q A_{11}}{A_{11} D_{11}-B_{11}^{2}} \\
& \frac{d^{3} u}{d x^{3}}=\frac{B_{11} d^{4} w}{A_{11} d x^{4}},
\end{aligned}
$$

and the B.C.s at $x=0, a$ are:

$$
\begin{aligned}
& w=0 \\
& N_{x x}=A_{11} \frac{d u}{d x}-B_{11} \frac{d^{2} w}{d x^{2}}=0
\end{aligned}
$$

$$
M_{x x}=B_{11} \frac{d u}{d x}-D_{11} \frac{d^{2} w}{d x^{2}}=0
$$

Equation (1) can be written as:

$$
\left(A_{11} D_{11}-B_{11}^{2}\right) \frac{d^{4} w}{d x^{4}}=q A_{11}
$$

By applying similitude theory, the resulting similarity conditions are:

$$
\lambda_{\mathrm{A}_{11}} \lambda_{\mathrm{D}_{11}} \lambda_{\mathrm{w}}=\lambda_{\mathrm{B}_{11}}^{2} \lambda_{\mathrm{w}}=\lambda_{\mathrm{A}_{11}} \lambda_{\mathrm{x}}^{4} \lambda_{\mathrm{q}},
$$

or

$$
\begin{aligned}
& \lambda_{\mathrm{A}_{11}} \lambda_{\mathrm{D}_{11}} \lambda_{\mathrm{w}}=\lambda_{\mathrm{B}_{11}}^{2}, \\
& \lambda_{\mathrm{w}} \lambda_{\mathrm{D}_{11}}=\lambda_{\mathrm{x}}^{4} \lambda_{\mathrm{q}} .
\end{aligned}
$$

Similarly from Eqs. (5), (6) and (7) we have:

$$
\begin{aligned}
& \lambda_{\mathrm{A}_{11}} \lambda_{\mathrm{u}} \lambda_{\mathrm{x}}=\lambda_{\mathrm{w}} \lambda_{\mathrm{B}_{11}}, \\
& \lambda_{\mathrm{B}_{11}} \lambda_{\mathrm{u}} \lambda_{\mathrm{x}}=\lambda_{\mathrm{w}} \lambda_{\mathrm{D}_{11}},
\end{aligned}
$$

The condition depicted by Eq. (13) can be obtained by combining Eqs. (10) and (12). So, Eqs. (10) through (12) denote the necessary conditions for complete similarity between the scale model and its prototype, as far as deflectional response is concerned.

Note that the similarity conditions, Eqs. (10)(12) are three, while the number of geometric and material parameters, cause parameter (load) and response parameters ( $u$ and $w$ ) is much larger than three. This means that there is freedom in designing models for a given prototype. In addition, if, in projocting the data of the model to predict the behavior of the prototype, all three scaling laws are used, then we have complete similarity. If only one (or two) scaling laws are used, then we have partial similarity.

For this particular application, experimental data was supplied by Professor Sierakowski ${ }^{2}$ for tests performed on beam plates. The total number of 
laminates used is ten. In Simitses ${ }^{43}$, some beam plates are considered as models and some as prototypes. Similitude theory is used and the results are compared to the test results of the prototypes (see Ref. 43 for details). Partial similarity is used in the comparison.

In addition to the above, similitude theory is employed in a case where experimental results do not exist. In this case, the theoretical results of the mor:al are treated as test data, then a scaling law (parial similarity) is used to predict the behavior of the prototype and the predictions are compared to the theoretical results of the prototype. If these $t: 0$ compare well, success has been achieved in designuing the model and in using similitude theory.

Consider a cross-ply laminated E-Glass/Ep , iy plate composed of 96 orthtropic layers $(0 / 90 / 0 / \ldots)$. is the prototype. We desire to find the maxim:m deflection of the nrototvoe by extrapolating the

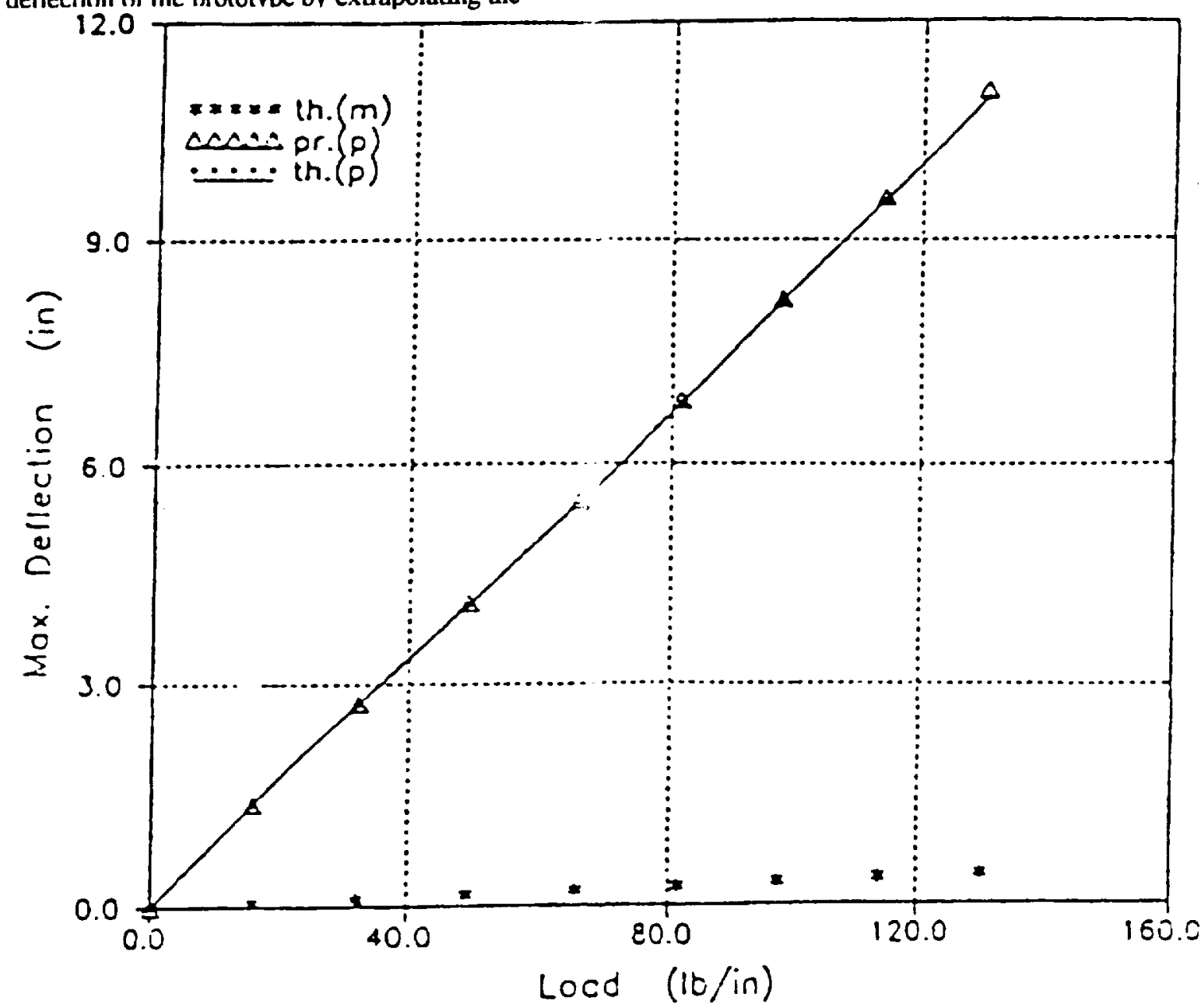

Fig. 1. Theoretical and predicted maximum deflections of prototype $\left(0^{\circ} / 90^{\circ} / 0^{\circ} \ldots\right)_{6}$ when the model is $\left(0 \% 90 \% 0^{\circ} \ldots\right)_{16}\left[\lambda_{E_{13}}=\lambda_{E_{22}}=\lambda_{v_{12}}=1 ; \lambda_{1}=18 ; \lambda_{b}=\lambda_{q}=16.92 ; \lambda_{b}=\lambda_{N}=6\right]$. pertinent values of a small scale model. The model has the same stacking sequence as the prototype but with a smaller number of layers. The prototype and its scale model have the following characteristics:

$$
\begin{aligned}
& \text { Prototype }\left(0(90 / 0 / \ldots)_{96}: a=90 \text { in. } \quad b=100\right. \text { in. } \\
& h=0.858 \text { in } \quad N=96 \text {, } \\
& \text { model }\left(0(90 / 0 / \ldots)_{16}: \quad a=5.0 \text { in } \quad b=6.139\right. \text { in. } \\
& h=0.143 \text { in. } N=16 \text {, } \\
& \text { scale factors: } \quad \lambda_{a}=18 \quad \lambda_{b}=16.29 \\
& \lambda_{h}=6 \quad \lambda_{N}=6 \text {. }
\end{aligned}
$$

In designing the model, we assume that it is made of the same material as the prototype and that $\lambda_{4}=\lambda_{b}$. By employing only the similarity condition of Eq. (11) (partial similarity), the results are plotted on Fig. 1. For details, see ref. 23. 


\section{Buckling and Vibrations of Plates}

Consider a simply supported, rectangular, symmetric, cross-ply laminated plate. The governing differential equation for buckling and vibration analyses is given by:

$$
\begin{gathered}
D_{11} w, x x x x+2 \bar{D}_{12} w, x x y y \\
=\rho w, D_{22} w, y y y y \\
\bar{N}_{x} w, x x
\end{gathered}
$$

For buckling alone the characteristic equation is:

$$
\begin{aligned}
\bar{N}_{X}= & \pi^{2}\left[D_{11}\left(\frac{m}{a}\right)^{2}+2 \bar{D}_{12}\left(\frac{n}{b}\right)^{2}\right. \\
& \left.+D_{22}\left(\frac{n}{b}\right)^{4}\left(\frac{a}{m}\right)^{2}\right]
\end{aligned}
$$

For free vibrations the characteristic equation is

$$
\rho \omega^{2}=\pi^{4}\left[D_{11}\left(\frac{m}{a}\right)^{4}+2 \bar{D}_{12}\left(\frac{m n}{a b}\right)^{2}+D_{22}\left(\frac{n}{b}\right)^{4}\right]
$$

By applying similitude theory to Eq. (14), we obtain:

$$
\lambda_{\rho} \lambda_{\omega}^{2}=\lambda_{D_{11}} \frac{\lambda_{m}^{4}}{\lambda_{a}^{4}}=\lambda_{D_{12}} \frac{\lambda_{m}^{2} \lambda_{n}^{2}}{\lambda_{a}^{2} \lambda_{b}^{2}}=\lambda_{D_{22}} \frac{\lambda_{n}^{4}}{\lambda_{b}^{b}}
$$

which yield the following scaling laws:

$$
\begin{aligned}
& \lambda_{\Omega}^{2}=\frac{\lambda_{\mathrm{D}_{11}}}{\lambda_{\mathrm{E}_{22}} \lambda_{\mathrm{h}}^{3}} \frac{\lambda_{\mathrm{m}}^{4}}{\lambda_{\mathrm{R}}^{4}}, \\
& \lambda_{\Omega}^{2}=\frac{\lambda_{\mathrm{D}_{12}}}{\lambda_{\mathrm{E}_{22}} \lambda_{\mathrm{h}}^{3}} \frac{\lambda_{\mathrm{m}}^{2} \lambda_{\mathrm{n}}^{2}}{\lambda_{\mathrm{R}}^{2}}, \\
& \lambda_{\Omega}^{2}=\frac{\lambda_{\mathrm{D}_{22}}}{\lambda_{\mathrm{E}_{22}} \lambda_{\mathrm{h}}^{3}} \lambda_{\mathrm{n}}^{4} \\
& \Omega^{2}=\frac{\mathrm{b}^{4} \omega^{2}}{\pi^{4}} \frac{\rho}{\mathrm{E}_{22} \mathrm{~h}^{3}}
\end{aligned}
$$

For details and results, see Refs. [25], [26]] and $[44]$.

\section{Application to Shell Configurations}

Complete similarity and partial similarity were applied to laminated cylindrical shell configurations. ${ }^{24,27,28}$ Details can be found in these references, but some basic equations and steps are presented, herein, for completeness. The buckling equation for a symmetric, laminated, cross-ply $\left(B_{i j}=\right.$ $D_{16}=D_{26}=A_{16}=A_{26}=0$ ), cylindrical shell (Ref. 45) is given by:

$$
\begin{aligned}
\mathrm{T}_{33} & +\frac{\left(2 \mathrm{~T}_{12} \mathrm{~T}_{13} \mathrm{~T}_{23}-\mathrm{T}_{11} \mathrm{~T}_{23}^{2}-\mathrm{T}_{22} \mathrm{~T}_{13}^{2}\right)}{\left(\mathrm{T}_{11} \mathrm{~T}_{22}-\mathrm{T}_{12}^{2}\right)} \\
& =-\overline{\mathrm{N}}_{\mathbf{x x}} \eta^{2}-\overline{\mathrm{N}}_{\mathrm{yy}} \xi^{2}
\end{aligned}
$$

where $\eta=\bar{m} \pi / L, \quad \xi=\bar{n} / R$,

$$
\begin{aligned}
& T_{11}=A_{11} \eta^{2}+A_{66} \xi^{2} \\
& T_{12}=\left(A_{12}+A_{66}\right) \xi \eta \\
& T_{13}=\frac{A_{12}}{R} \eta
\end{aligned}
$$

$$
\begin{aligned}
& \mathrm{T}_{22}=\mathrm{A}_{22} \xi^{2}+\mathrm{A}_{66} \eta^{2} \\
& \mathrm{~T}_{23}=\frac{\mathbf{A}_{22}}{\mathrm{R}} \xi
\end{aligned}
$$

$\mathrm{T}_{33}=\mathrm{D}_{11} \eta^{4}+2 \overline{\mathrm{D}}_{12} \xi^{2} \eta^{2}+\mathrm{D}_{22} \xi^{4}+\frac{\mathrm{A}_{22}}{\mathrm{R}^{2}}$

and

$$
\bar{D}_{12}=D_{12}+2 D_{66}
$$

The lowest eigenvalue corresponds to the buckling load, and minimization with respect to integer values of $\mathrm{m}$ and $\mathrm{n}$ yields the critical load.

Equation (20) represents the buckling response of both prototype and its models. Applying similitude theory to the preceding equation, Eq. (20) yields the following scaling laws for symmetric, cross-ply, laminated cylinders:

$$
\left(\frac{\lambda_{\eta}}{\lambda_{\xi}}\right)^{4}=\frac{\lambda_{\mathrm{A}_{22}}}{\lambda_{\mathrm{A}_{11}}}
$$




$$
\begin{aligned}
& \left(\frac{\lambda_{\eta}}{\lambda_{\xi}}\right)^{2}=\frac{\lambda_{\mathrm{A}_{22}}}{\lambda_{\mathrm{A}_{66}}} \\
& \left(\frac{\lambda_{\eta}}{\lambda_{\xi}}\right)^{2}=\frac{\lambda_{\mathrm{A}_{66}}}{\lambda_{\mathrm{A}_{11}}} \\
& \left(\frac{\lambda_{\eta}}{\lambda_{\xi}}\right)^{2}=\frac{\lambda_{\mathrm{A}_{66}}}{\lambda_{\mathrm{A}_{11}} \lambda_{\mathrm{A}_{66}}} \\
& \lambda_{\Psi}=\lambda_{\eta}^{4} \lambda_{\mathrm{D}_{11}} \\
& \lambda_{\Psi}=\lambda_{\mathrm{D}_{12}} \lambda_{\eta}^{2} \lambda_{\xi}^{2} \\
& \lambda_{\Psi}=\lambda_{\mathrm{D}_{22}} \lambda_{\xi}^{4} \\
& \lambda_{\Psi}=\frac{\lambda_{\mathrm{A}_{22}}}{\lambda_{\mathrm{R}}^{2}} \\
& \lambda_{\Psi}=\frac{\lambda_{\mathrm{A}_{12}}^{2}}{\lambda_{\mathrm{A}_{11}} \lambda_{\mathrm{R}}^{2}}
\end{aligned}
$$

where

$$
\begin{aligned}
& \Psi=-\overline{\mathrm{N}}_{\mathrm{xx}} \eta^{2},-\overline{\mathrm{N}}_{\mathrm{yy}} \xi^{2}, \text { or }-(\mathrm{pR} / 2)\left(\eta^{2}+2 \xi^{2}\right), \\
& \text { and } \overline{\mathrm{A}}_{12}=\mathrm{A}_{12}+\mathrm{A}_{66}, \\
& \overline{\mathrm{D}}_{12}=\mathrm{D}_{12}+2 \mathrm{D}_{66} .
\end{aligned}
$$

The nine scaling laws, Eqs. (22)-(30) are the necessary scaling laws for cross-ply laminated cylindrical shells for axial compression, lateral pressure, and hydrostatic pressure. The conditions that represent structural geometries and mode shapes, Eqs. (22)-(25) are the necessary scaling laws for symmetric cross-ply laminated cylinders regardless of the destabilizing load.

As is apparent, the scaling laws are arranged in the form of different scale factors for each load case ( $\psi$ ). It should be pointed out that the presented form of arranging the scaling laws is not unique. However, previous experience of establishing scaling laws ${ }^{25}$ strongly recommends this type of representation.

\section{Scaling Laws for Lateral Pressure Load}

For the case of a cylinder subjected to lateral pressure p, $N_{y y}=p R$ and Eqs. (26)-(30) assume the following form:

$$
\begin{aligned}
& \lambda_{\mathrm{K}_{\mathrm{yy}}}=\frac{\lambda_{\eta}^{4}}{\lambda_{\xi}^{2}} \lambda_{\mathrm{L}}^{2} \\
& \lambda_{\mathrm{K}_{\mathrm{yy}}}=\frac{\lambda \overline{\mathrm{D}}_{12}}{\lambda_{\mathrm{D}_{11}}} \lambda_{\eta}^{2} \lambda_{\mathrm{L}}^{2} \\
& \lambda_{\mathrm{K}_{\mathrm{yy}}}=\frac{\lambda_{\mathrm{A}_{22}}}{\lambda_{\mathrm{D}_{11}}} \frac{\lambda_{\mathrm{L}}^{2}}{\lambda_{\mathrm{R}}^{2} \lambda_{\xi}^{2}} \\
& \lambda_{\mathrm{K}_{\mathrm{yy}}}=\frac{\lambda_{\mathrm{A}_{12}}^{2}}{\lambda_{\mathrm{A}_{11}} \lambda_{\mathrm{D}_{11}}} \frac{\lambda_{\mathrm{L}}^{2}}{\lambda_{\mathrm{R}}^{2} \lambda_{\xi}^{2}} \\
& \lambda_{\mathrm{K}_{\mathrm{yy}}}=\frac{\lambda_{\mathrm{D}_{22}}}{\lambda_{\mathrm{D}_{11}}} \lambda_{\xi}^{2} \lambda_{\mathrm{L}}^{2}
\end{aligned}
$$

where $K_{y y}=-\bar{N}_{y y} L^{2} / \pi^{2} D_{11}, \quad \bar{A}_{12}$, and $\bar{D}_{12}$, have already been defined, and $\lambda x_{i}=x_{i p} / x_{i_{m}}$ denotes the scale factor of parameter $x_{i}$

Parenthetical remarks: For the case of lateral pressure $\psi=-\overline{\mathbf{N}}_{\mathbf{y y}} \xi^{2}$. Therefore,

$\lambda_{\Psi}=\lambda \bar{N}_{y y} \lambda_{\xi}^{2}$. Similarly, from the definition of $\mathrm{K}_{\mathrm{yy}}\left(\mathrm{K}_{\mathrm{yy}}=\overline{\mathrm{N}}_{\mathrm{yy}} \mathrm{L}^{2} / \pi^{2} \mathrm{D}_{11}\right)$, one can write $\lambda_{K_{y y}}=-\lambda \bar{N}_{y y} \lambda_{L}^{2} / \lambda D_{11}$. Use of these two expressions in Eq. (26) yields Eq. (31). In a similar manner one can derive Eqs. (32)-(35).

Equations (31)-(35) are the necessary scaling laws for symmetric, cross-ply, laminated cylinders subjected to uniform lateral pressure.

The interested reader is referred to Refs. 24 and 27 for results with primarily partial similarity with 
distortion in number of plies, stacking sequence and cylinder length, radius and thickness. Distortion here means that prototype and model have different parameters (as mentioned above).

\section{Discussion}

It has been demonstrated through the studies reported herein, that structural similitude is a powerful tool in minimizing the need for full scale and large component testing of structural systems. Future work should include the study of systems that exhibit imperfection sensitivity, extension to sandwich configurations and validation of the process through an experimental program for laminated plates and shells as well as beam plates, plates and shells of sandwich construction.

Through this review, the authors have demonstrated a procedure that can be used in designing small scale and easily testable models to predict the behavior of large prototypes through the use of scaling laws. These laws are based on the premise that both model and prototype are governed by the same field equations and that the systems behave in a linearly elastic manner and they are free of damage (delaminations, fiber breaks, matrix microcracking, etc.). This last premise guarantees that no size effects are present.

\section{Acknowledgement}

Work was supported by NASA Langley Research Center, under Grant No. NASA NAG-1-2071. The financial support provided by NASA is gratefully acknowledged

\section{References}

1. McDougal, R.L., Private communications, Structural Division, the Lockheed-Georgia Company, Marietta, GA, 1987.

2. Kline, S.J., Similitude and Approximation Theory. McGraw-Hill, NY, 1965.

3. Rayleigh, Lord, "The Principle of Similitude," Nature, 95, 66-68, 1915.

4. Macagno, E.O., "Historico-Critical Review of Dimensional Analysis," J. Franklin Inst., Vol. 292, No. 6, pp. 391-402, 1971.
5. Goodier, J.N. and Thomson, W.T., "Applicability of Similarity Principles to Structural Models," NACA Tech. Note 993, 1944.

6. Goodier, J.N., "Dimensional Analysis," Handbook of Experimental Stress Analysis (Edited by M. Hetenyi), pp. 1035-1045, John Wiley \& Sons, NY, 1950.

7. Murphy, G., Similitude in Engineering, Ronald Press, New York, 1950.

8. Langhaar, H.L., Dimensionless Analysis and Theory of Models, John Wiley \& Sons, New York, 1951 .

9. Charlton, T.M., Model Analysis of Structures, John Wiley \& Sons, New York, 1954.

10. Pankhust, R.C., Dimensional Analysis and Scale Factors, Chapman \& Hall, London, Reinhold, New York, 1964.

11. Gukhman, A.A., Introduction to the Theory of Similarity, Academic Press, New York, 1965.

12. Szucs, E., Similitude and Modelling, Elsevier, NY, 1980.

13. Singer, J., Arbocz, I. And Weller, T., Buckling Experiments, John Wiley and Sons, Chickester, England, 1997.

14. Ezra, A.A., "Similitude Requirements for Scale Model Determination of Shell Buckling under Impulsive Pressure," NASA TN D-1510, pp. 661670, 1962.

15. Morgen, G.W., Scaling Techniques for Orthotropic Cylindrical Aerospace Structures," Proceedings of AIAA $5^{\text {th }}$ Structures and Materials Conference, Pal Springs, pp. 333-343, April 1964.

16. Soedel, W., "Similitude Approximations for Vibrating Thin Shells," J. Acoustical Saciety of America, Vol. 49, No. 5, pp. 1535-41, 1971.

17. Morton, J., "Scaling of Impact Laaded Carbon Fiber Composites," ALAA Journal, Vol. 26, No. 8 , pp. 989-994, 1988.

18. Qian, Y., Swanson, S.R, Nuismer, RJ. and Bucinell, RB., "An Experimental Study of Scaling Rules for Impact Damage in Fiber Composites," $J$. Composite Materials, Vol. 24, No. 5, pp. 559-570, 1090 
19. Shih, C., Chen, J.C. and Garba, J., "Verification of Large Beam-Type Space Structures," NASA Report No. 87-22712, 1987.

20. Letchworth, R, McGowan, P.E. and Gronet, J.J., "Space Station: A Focus for the Development of Structural Dynamics Scale Model for Large Flexible Space Structures," presented at AIAA/ASME/ASCE/AHS $29^{\text {th }}$ SDM Conference (not included in Proceedings), Williamsburg, VA, April 18-20, 1988.

21. Hsu, C.S., Griffin, J.H. and Bielak, J., "How Gravity and Joint Scaling Affect Dynamic Response," ALAA Journal, Vol. 27, No. 9, pp. 1280-87, 1989.

22. McGowan, P.E., Edighoffer, H.E. and Wallace, J.W., "Development of an Experimental Space Station Model for Structural Dynamics Researches," NASA Technical Memorandum $102601,1990$.

23. Simitses, G.J. and Rezacepazhand, J., "Structural Similitude for Laminated Structures," $J$. Composites Engineering, Vol. 3, Nos. 7-8, pp.; 751-765, 1993.

24. Rezacepazhand, J., Simitses, G.J. and Starnes, J.H. Jr., "Scale Models for Laminated Cylindrical Shells Subjected to Axial Compression," Composite Structures, Vol. 34, No. 4, pp. 371-379, 1996.

25. Rezacepazhand, J., Simitses, G.J. and Starnes, J.H., Jr., "Design of Scaled Down Models for Stability of Laminated Plates," ALAA Joumal, Vol. 33, No. 3, pp. 515-519, 1995.

26. Simitses, G.J. and Rezaeepazhand, J., "Structural Similitude and Scaling Laws for Buckling of Cross-Ply Laminated Plates," J. Thermoplastic Composite Materials, Vol. 8, pp. 240-251, 1995.

27. Simitses, G.J., Rezaeepezhand, J. and Sierakowski, R.L., "Scaled Models for Laminated Cylindrical Shells Subjected to External Pressure," Mechanics of Comp. Materials \& Structures, Vol. 4, pp. 267280, 1997.

28. Rezaeepazhand, J. and Simitses, G.J., "Structural Similitude for Vibration Response of Laminated Cylindrical Shells with Double Curvature," Composites, Part B, Vol. 28B, pp. 195-200, 1997.
29. Chouchaoui, C.S. and Ochoa, O.O., "Similitude Study for a Laminated Cylindrical Tube Under Tensile, Torsion, Bending, Internal and External Pressure, Part I: Governing Equations," Composite Structures, Vol. 44, pp. 221-229, 1999.

30. Chouchaoui, C.S., Parks, P. and Ochoa, O.O., "Similitude Study for a Laminated Cylindrical Tube Under Tensile, Torsion, Bending, Internal and External Pressure. Part II: Scale Models," Composite Structures, Vol. 44, pp. 231-236, 1999.

31. Jackson, K.E. (compiler), "Workshop on Scaling Effects in Composite Materials and Structures," NASA Conference Publication 3271, July 1994.

32. Bazant, Z.P. and Rajapakse, Y.D.S. (editors), Fracture Scaling, Kluwer Academic Publishers, Dordrecht, 1999.

33. Jackson, K.E. and Kellas, S., "Sub-Ply Level Scaling Approach Investigated for Graphite-Epoxy Composite Beam-Columns," NASA CP 3271 (Ref. 29), pp. 19-36, 1994.

34. Camponeschi, G., "The Effects of Specimen Scale on the Compression Strength of Composite Materials," NASA CP 3271, pp. 81-99, 1994.

35. Johnson, D.P., Morton, J,, Kellas, S. and Jackson, K.E., "Scaling Effects in the Tensile and Flexure Response of Laminated Composite Coupons," NASA CP 3271, pp. 265-282, 1994.

36. Grimes, G.C., "Experimental Observations of Scale Effects on Bonded and Bolted Joints in Composite Structures," NASA CP 3271, pp. 57-80, 1994.

37. O'Brien, T.K., "Damage and Strength of Composite Materials: Trends, Predictions and Challenges," NASA CP 3271, pp. 145-160, 1994.

38. Greszczuk, L.B., "Private Communication" September 23, 1999.

39. Daniel, I.M. and Hsiao, H.M., "Is There a Thickness Effect on Compressive Strength of Unnotched Composite Laminates?" in Fracture Scaling, Z.P. Bazant and Y.D.S. Rajapakse (editors), Klumer Academic Publishers, pp. 143158, 1999.

40. Jackson, K.E., "Scaling Effects in the Static and Dynamic Response of Graphite-Epoxy BeamColumns," NASA TM 102697, July 1990. 
41. Ashton, J.E. and Whitney, J.M., Theory of Laminated Plates, Technomic Publ., Stamford, CT 1970.

42. Sierakowski, R.L., "Private Communication," Ohio State University, Columbus, OH, 1994.

43. Simitses, G.J., "Structural Similitude for Flat Laminated Surfaces," Composite Structures, accepted for publication, 1999.

44. Rezaeepazhand, J., Simitses, G.J. and Starnes, J.H., Jr., "Use of Scaled Down Models for Predicting Vibration Response of Laminated Plates," Composite Structures, Vo. 30, pp. 419-426, 1995.

45. Jones, R.M. and Morgan, H.S., "Buckling and Vibration of Cross-Ply Laminated Circular Cylindrical Shells," AIAA Journal, Vol. 13, No. 5, pp. 664-671, 1975 . 
